\title{
احكام الاجارة المنتهية بالتمليك
}

Doi: 10.23918/ilic2020.06

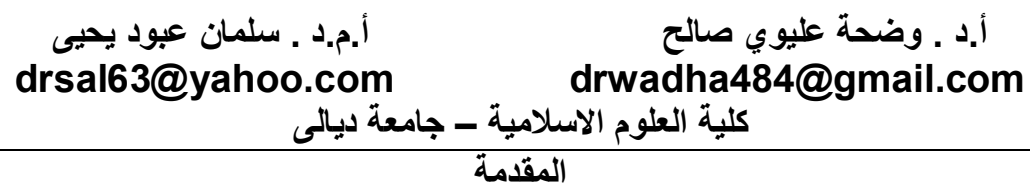

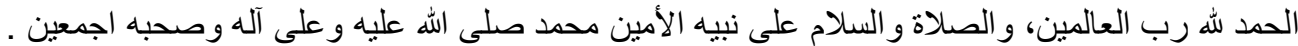

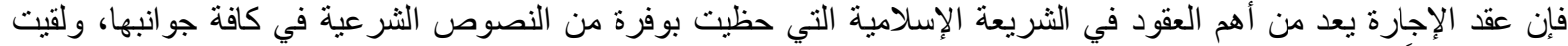

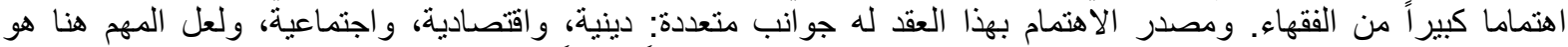

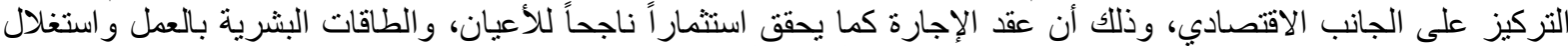

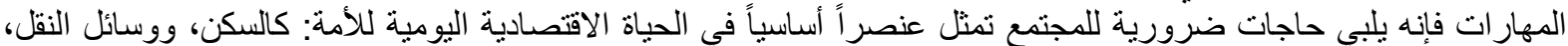

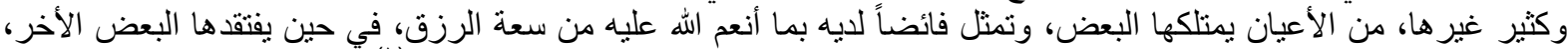

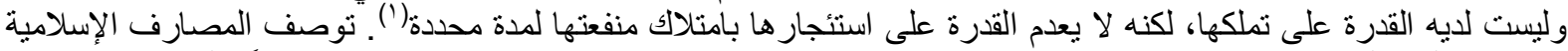

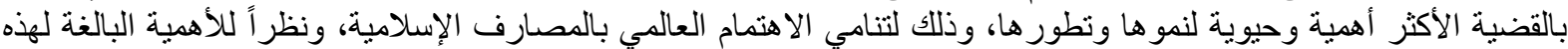

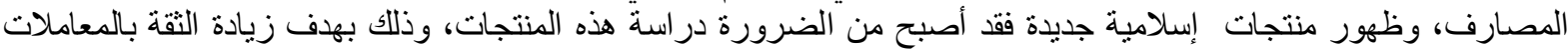

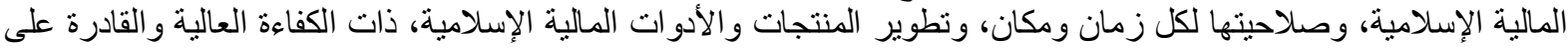
مواجهة الأزمات وتفعيل النشاط الاقتصادي.

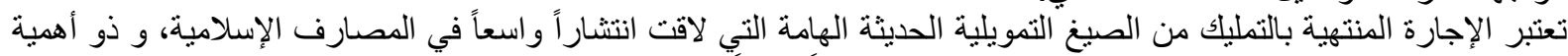

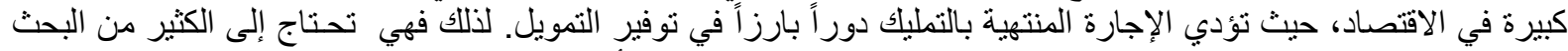

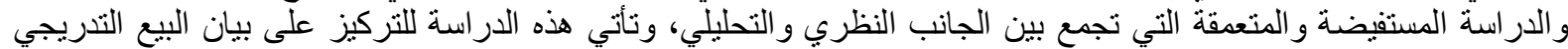

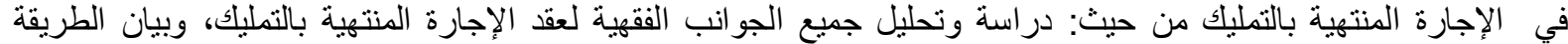

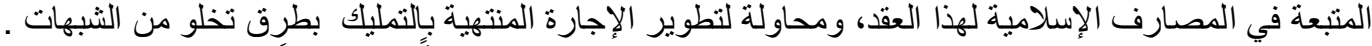

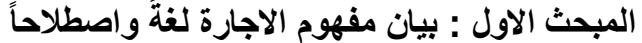

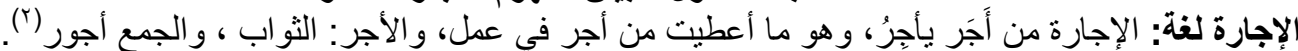

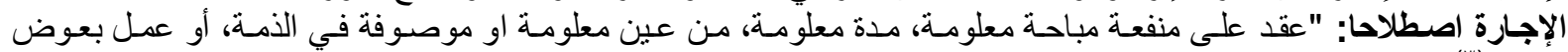

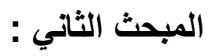

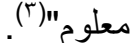

اولاً : مشروعية الإجارة

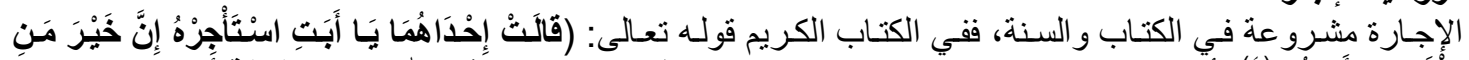

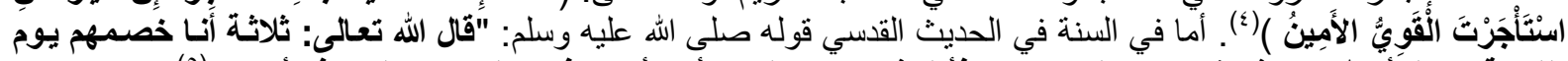

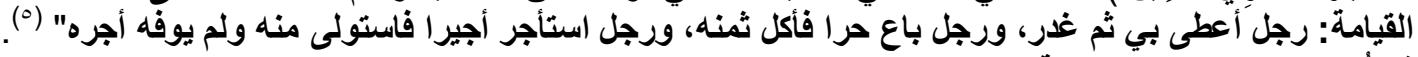

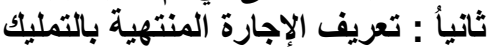

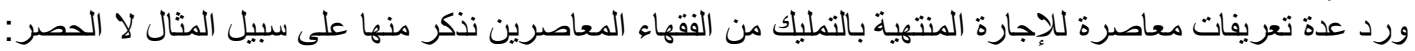
ا - " أن بتفق الطرفان على إجارة شيء لمدة معينة بأجرة معلومة ـ قد تزيد على أجرة المثل -, على أن تنتهي بتمليك العين

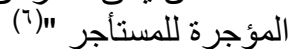
r- " إجارة بقترن بها الو عد بتمليك العين المؤجرة إلى المستأجر في نهاية مدة الإجارة أوفي أثنائها, ويتم التمليك بأحد الطرق

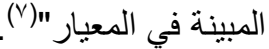

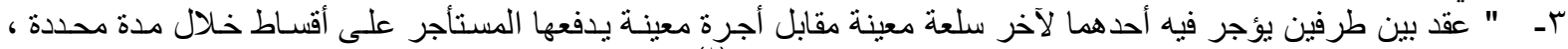

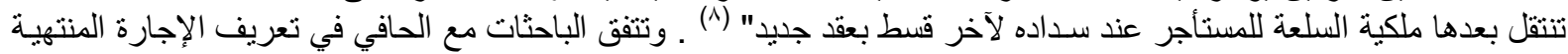
بالتمليك . أسماء عقد الإجارة المنتهية بالتمليك : يطلق على هذا العقد عدة إطلاقات منها (9) الإنطاء

$$
\begin{aligned}
& \text { (') أبو سليمان، عبد الو هاب إبر اهيم، عقد الإجارة من مصادر التمويل الإسلامي دراسة فقهية مقارنة، المعهد الإسلامي للبحوث و التدريب، ط؟، .... ب، } \\
& \text { صالا. (1) }
\end{aligned}
$$

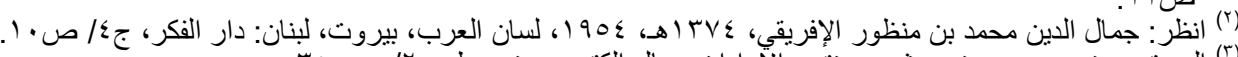

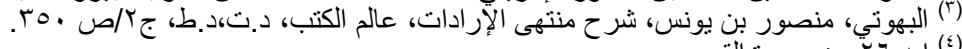

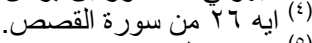

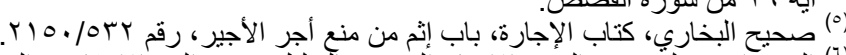

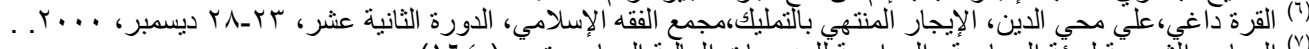

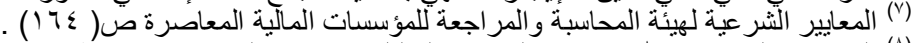

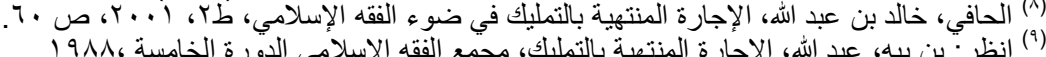




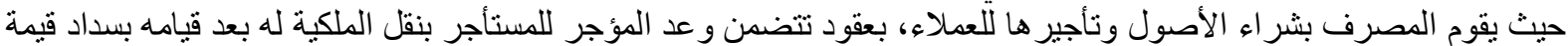

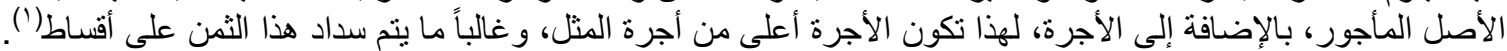

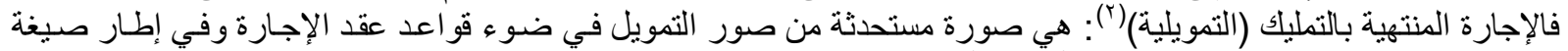

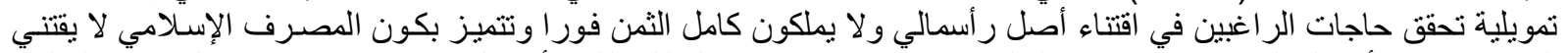

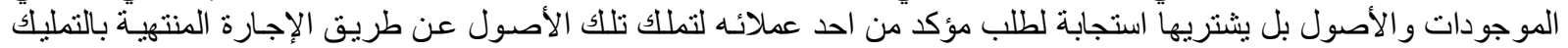

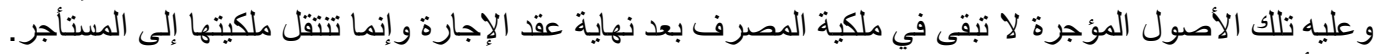

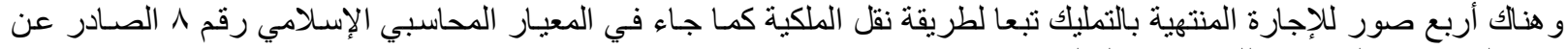

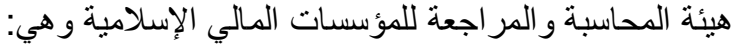

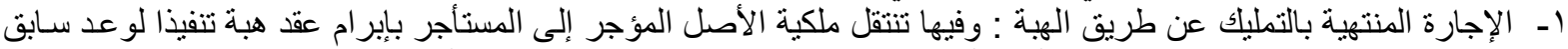

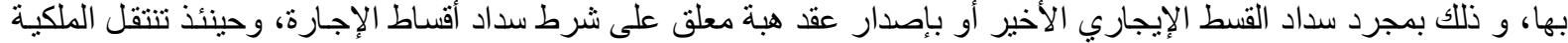

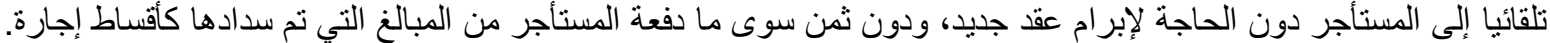

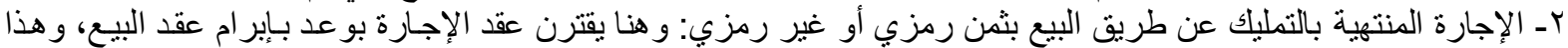

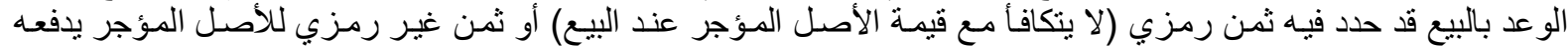

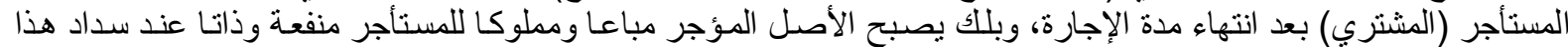
الثمن المتفق عليه.

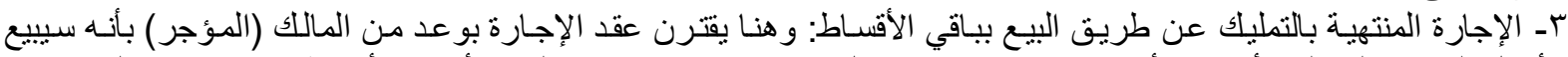

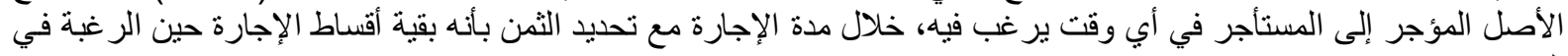
الثر اء.

ع- الإجارة المنتهية بالتمليك عن طريق البيع التدريجي: و هنا يقترن عقد الإجارة بوعد من المالك (المؤجر) بأنه سيبيع للمستأجر

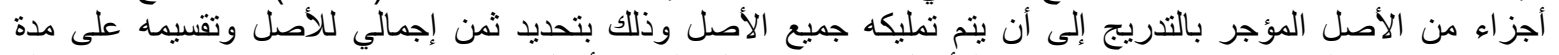

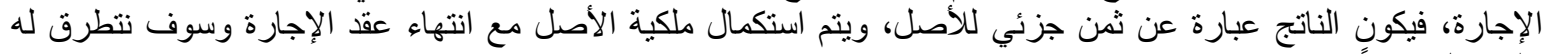

\section{المبحث الثالث : التخريجات الفقهية للإجارة المنتهية بالتمليك}

بالتفصيل لاحقاً.

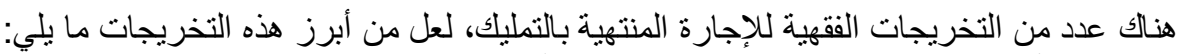

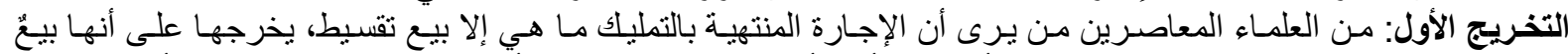

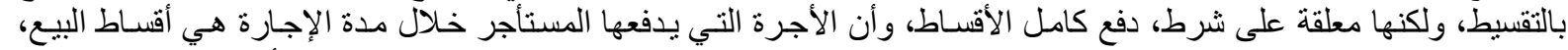

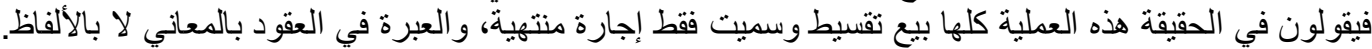

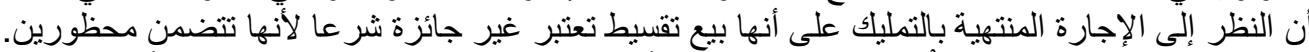

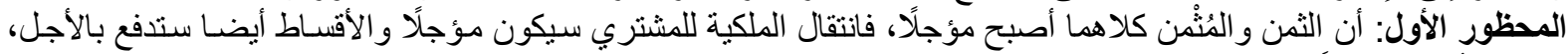

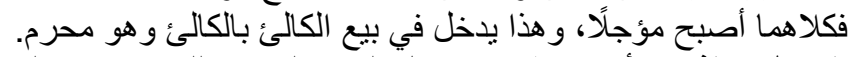

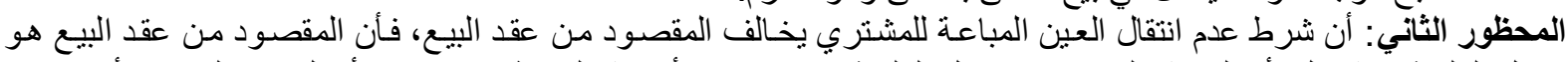

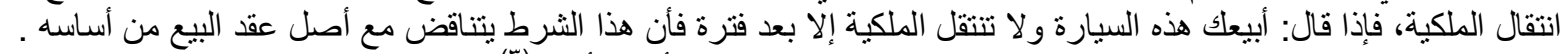

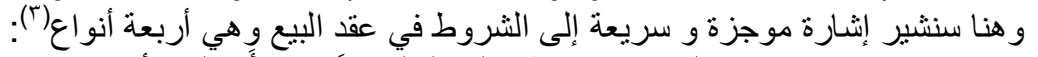

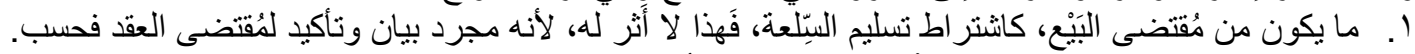

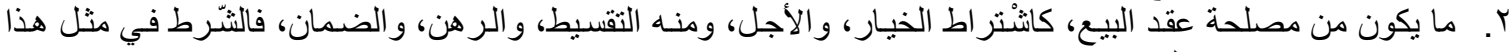

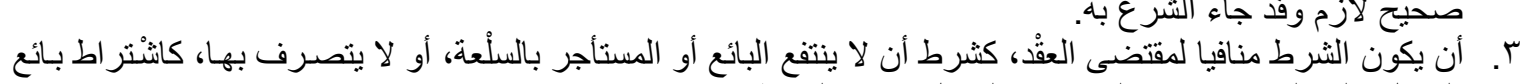

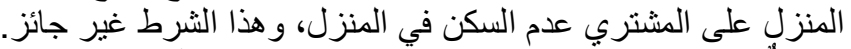

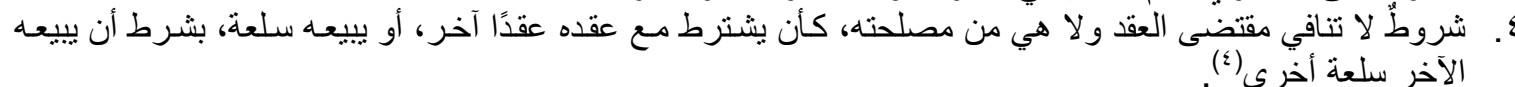

(') أبو الهيجاء، الياس ، تطوير آلبات التمويل بالمشاركة في المصارف الإسلامية- دراسة حالة الأردن- رسالة دكتوراه غير منشورة، جامعة البرموك

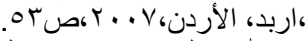

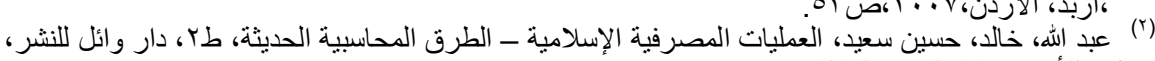

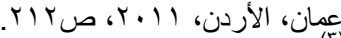

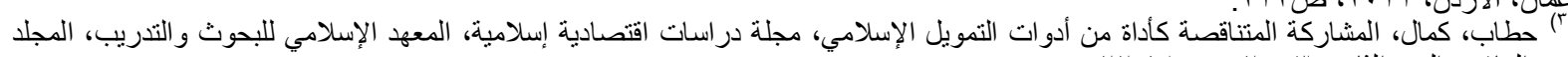

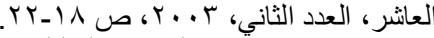

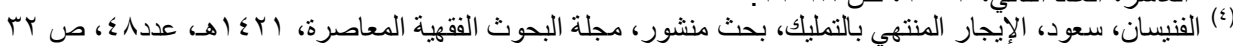


و على هذا فنقول في الإجارة المنتهية بالتمليك: لو قلنا أنها بيع تقسيط لكن ملكية السلعة لا تنتقل إلى المشتري إلا بعد الانتهاء من

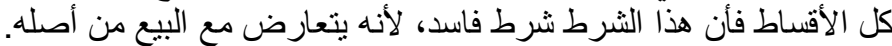

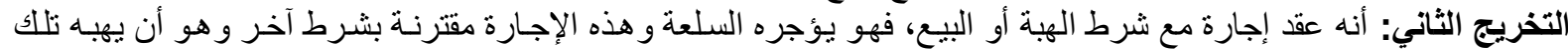

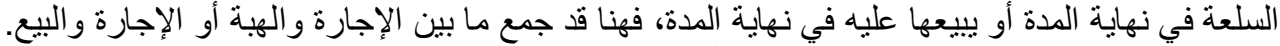
إن هذا التخريج يتضمن اشتر اط عقد في عقد، أي اجتمع عقدين في عقد، هما عقد الإجارة و عقد الهبة، أو عقد الإجارة و عقد البيع، وقد اختلف الفقهاء في هذه المسألة على رلئ رأيين.

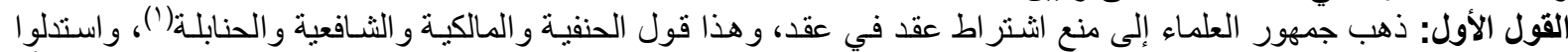

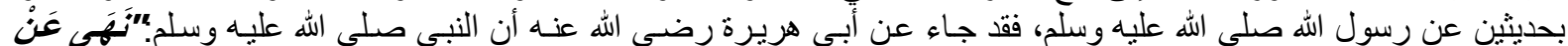

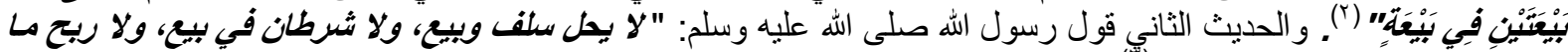

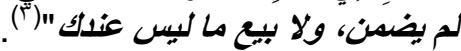

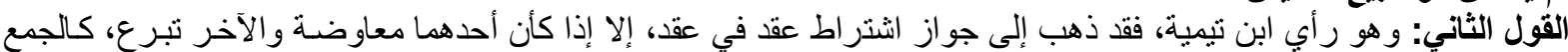

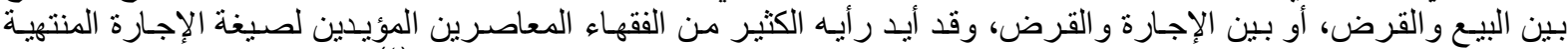

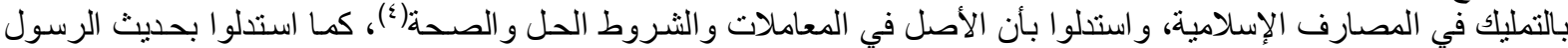

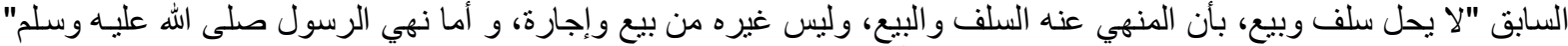

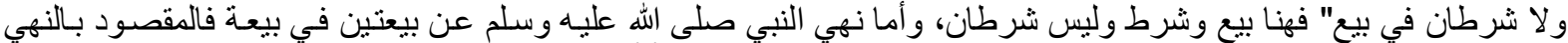

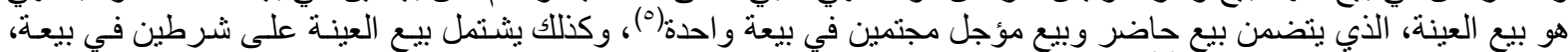

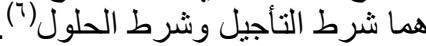

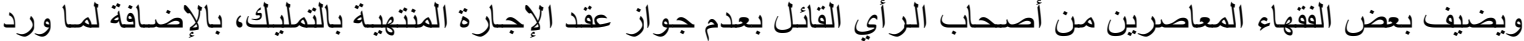

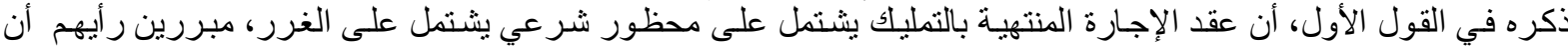

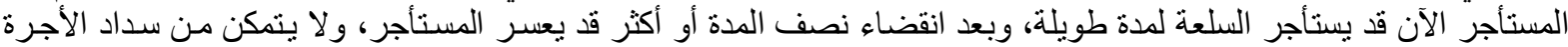

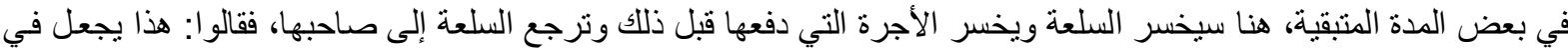

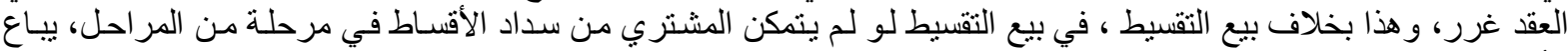

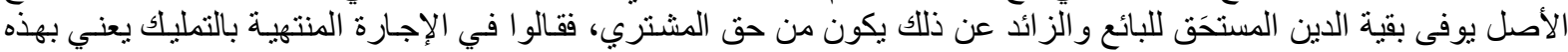

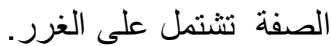

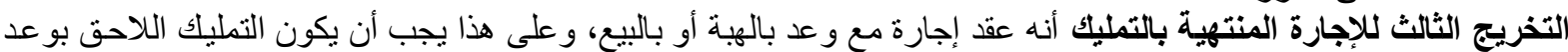

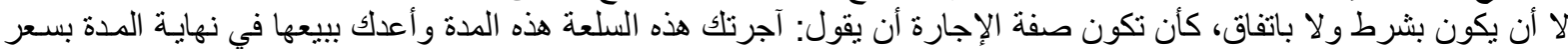

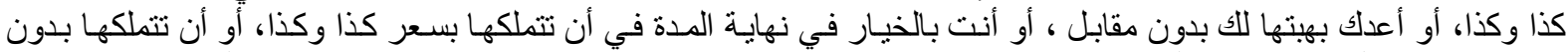

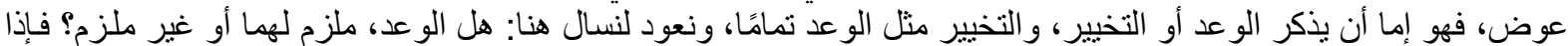

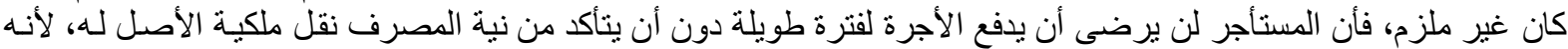

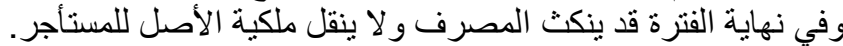
أما إذا كأن الو عد ملزمة يجعل الو عد الملزمٍ في حكم البيع المؤجل، فيكون الثمن و المثمن مؤجلا الدفع، فيؤدي إلى صورة الكالئ بالكالىئ. و على هذا التخريج يجب أن تطبق أحكام الإجارة في فترة الإجارة، ثم في نهاية المدة يكون للطرفين الحق في أنشاء عقد جديد، إما عقد بيع أو عقد هبة. و أخير انورد رأي مجلس مجمع الفقه الإسلامي الدولي المنبثق عن منظمة المؤتمر الإسلامي في دورته الثانية الثانية عشرة بالرياض في

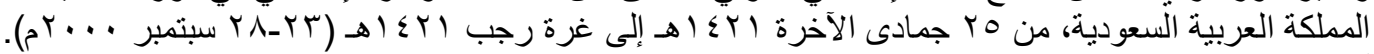

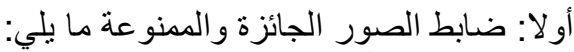
أ -ضابط المنع: أن يرد عقدان مختلفان في وقةت والجة واحد، على عين واحدة، في زمن واحد.

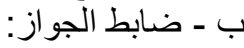
أ -وجود عقديط منفيطلين يستقل كل منهما عن الآخر ، زمانا بحيث يكون إبرام عقد البيع بعد عقد الإجارة، أو وجود و وعد بالتمليك

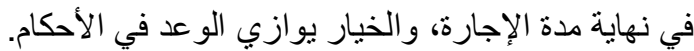
r أن تكون الإجارة فعلية، وليست ساترة الإن اللبيع.

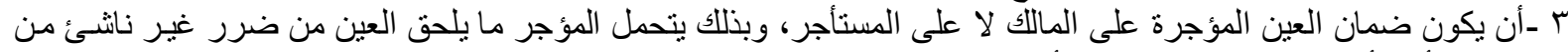

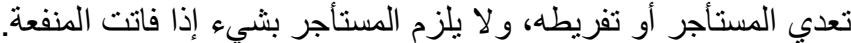
ــ ـإذا اشتمل العقد على تأمين العين المؤجرة، فيجب أن يكون التأمين تعاونيًا إسـلاميًا لا تجاريًا، ويتحمله المالك المؤجر وليس المستأجر: أن تطبق على عقد الإجارة المنتهية بالتمليك أحكام الإجارة طوال مدة الإجارة وأحكام البيع عند تملك العين.

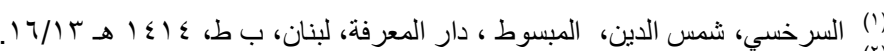

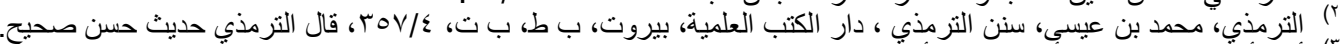

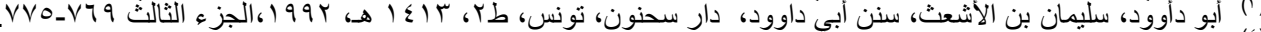

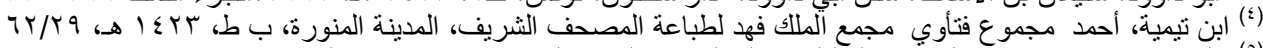

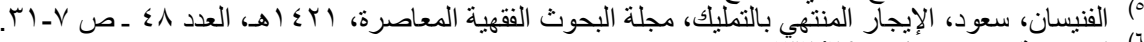

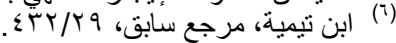


7 - 1 -تكون نفقات الصيانة غير التشغيلية على المؤجر لا على المستأجر طو ال مدة الإجارة.

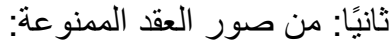

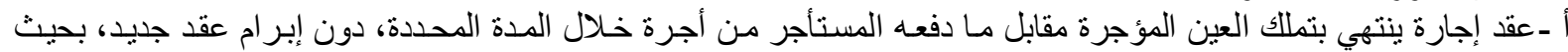
تقنلب الإجارة في نهاية المدة بيعًا تلقائيًا.

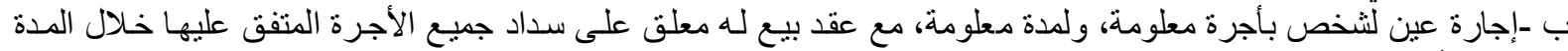

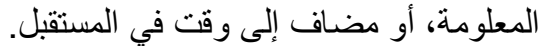

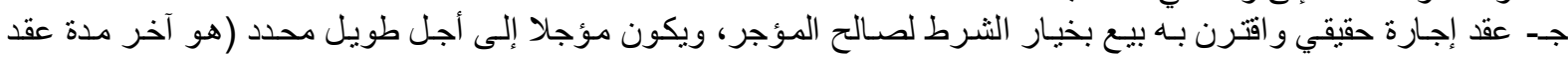
الإيجار). وهذا مأ تضمنته الفتاوى و القرار ات الصادرة من هيئات علمية، ومنها هيئة كبار العلماء بالمملكة العربية السعودية.

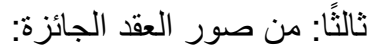

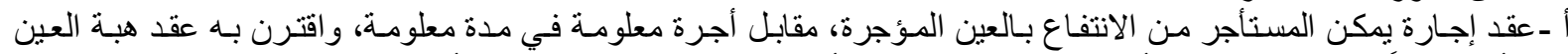

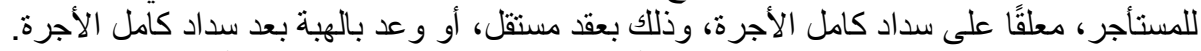

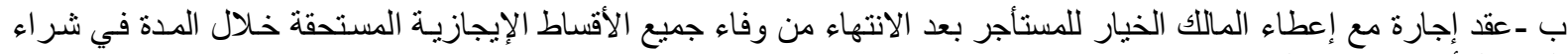

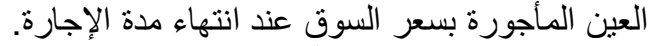

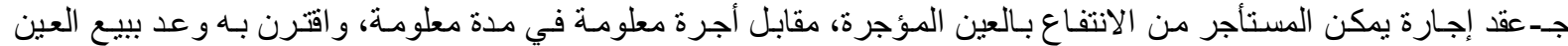

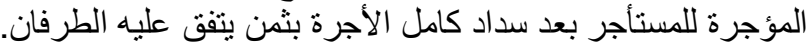

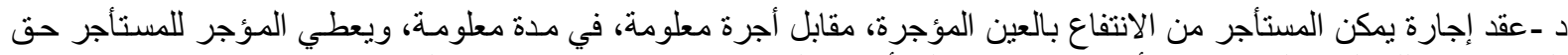

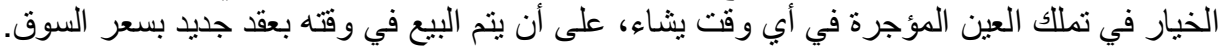

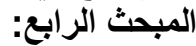

اولاً : - الطرق المتبعة في المصارف الإسلامية في تطبيق صيغة الإجارة المنتهية بالتمليك

الإجارة المنتهية بالتمليك كما تجريها المصارف الإسلامية لا تخرج عن واحدة من الصور الثناث الأتية: الصورة الأولى: أن تكون عقد إجارة مقرونة بهبة للسلعة محل العقد، بحيث تنتقل ملكيـة السلعة محل العقد في نهايـة المدة من الإنة

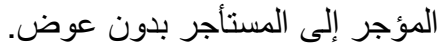

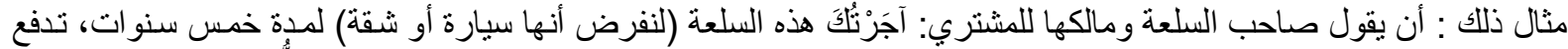

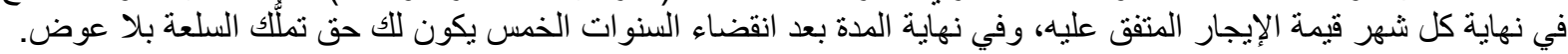

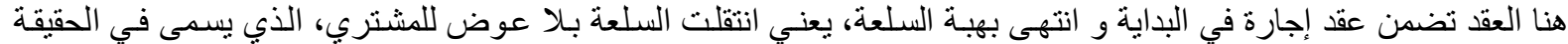
مستأجر ا.

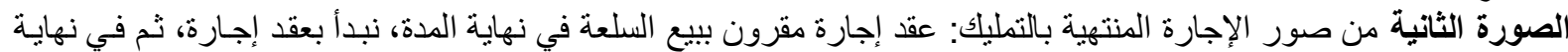

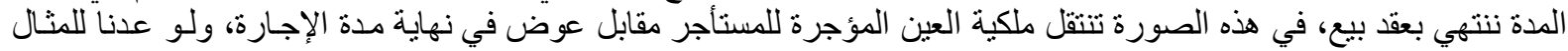

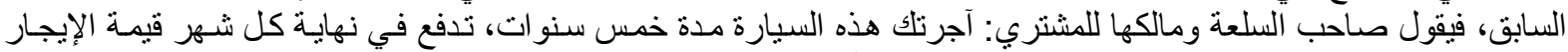

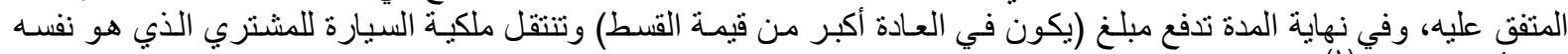

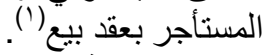

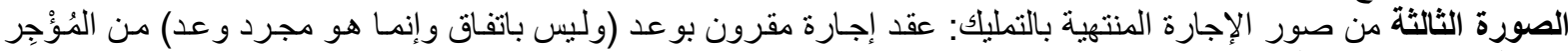

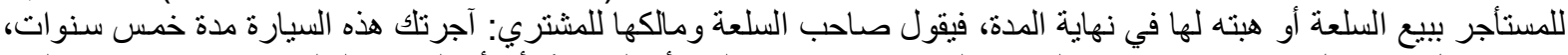

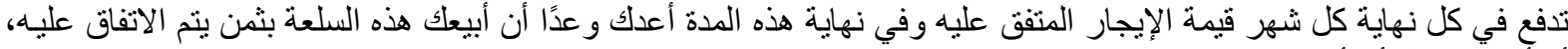

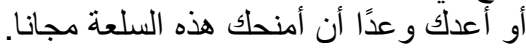

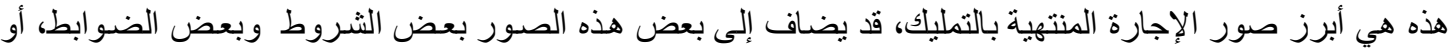

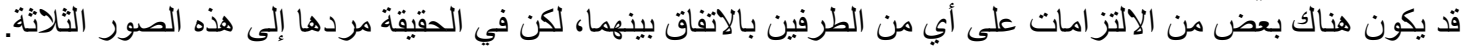

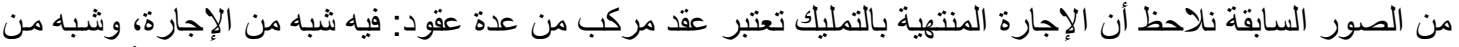

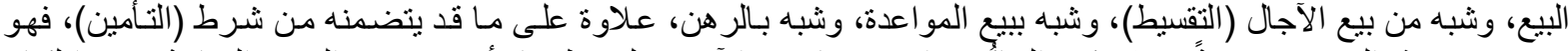

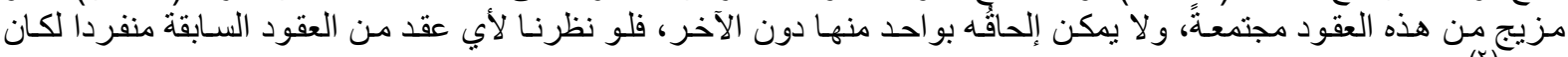
جائز إ) (ب)

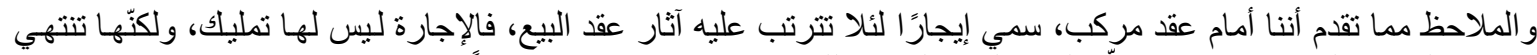

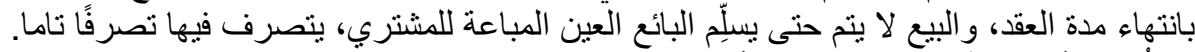

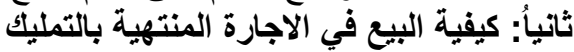

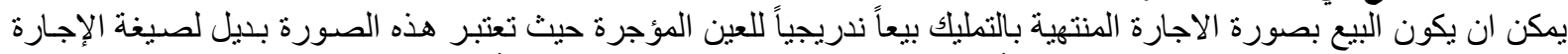

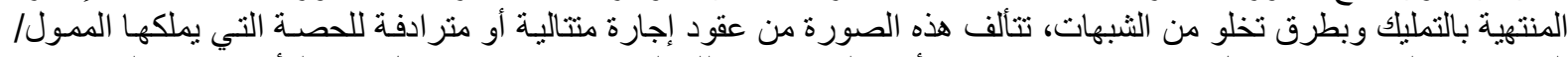

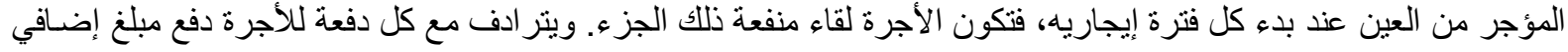

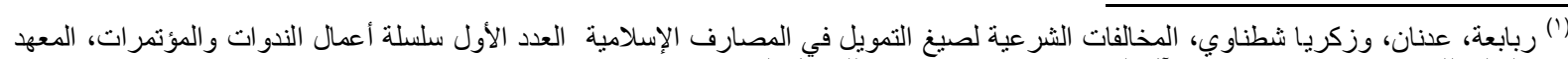

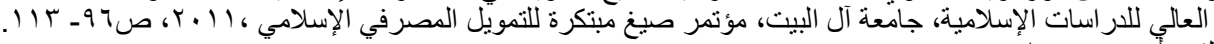
(؟) الفنيسأن، مرجع سابق. 


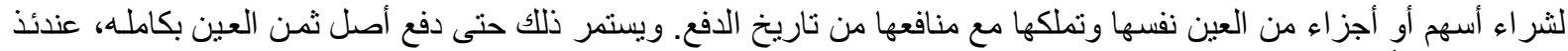

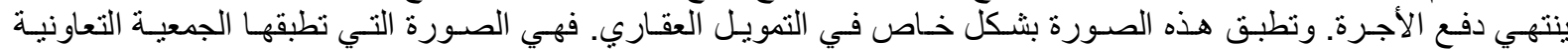

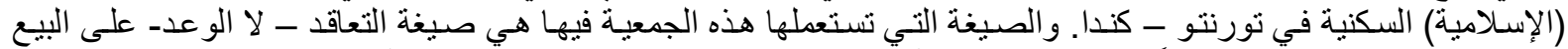

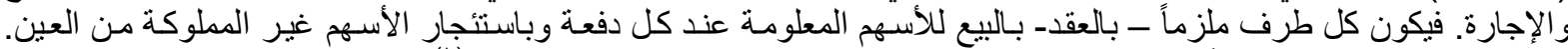

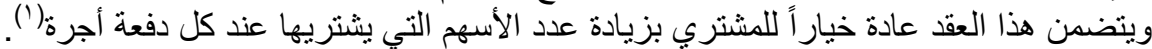

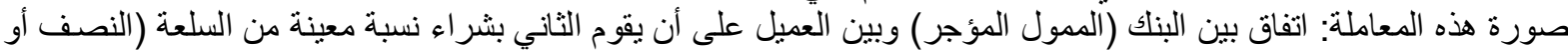

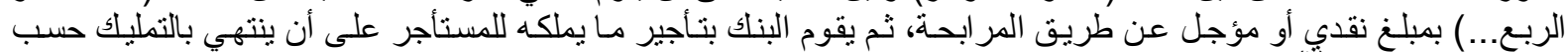

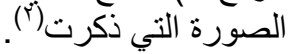

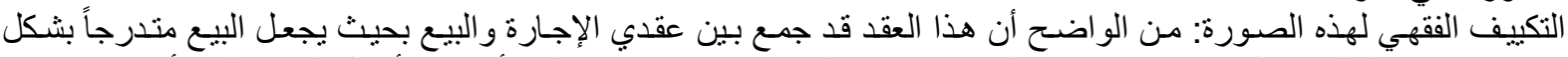

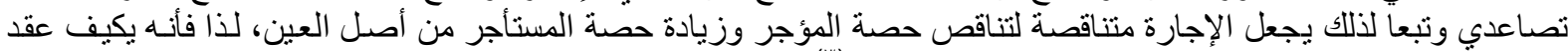

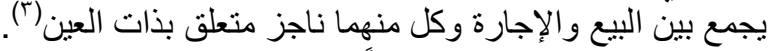

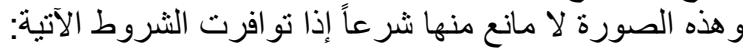

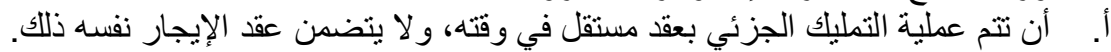

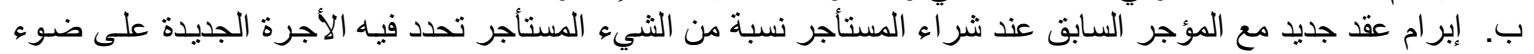
تغير نسبة الملكية. ت. هذه الصورة لا تحتوي على لفئ بيع ما لا يملك أو لا يقبض، لأن السلعة المؤجرة في ملك البائع وضمانه، وقد قبضهها فعلاً

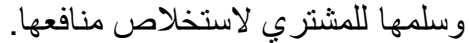

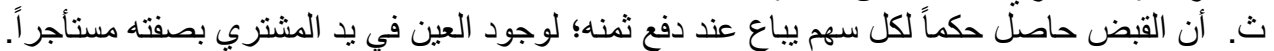

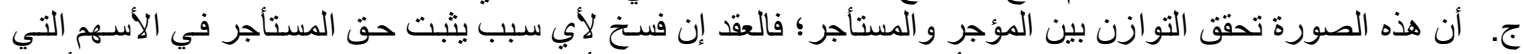

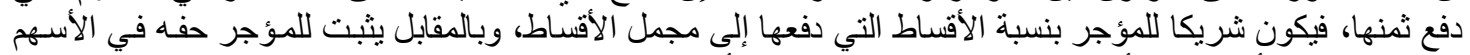

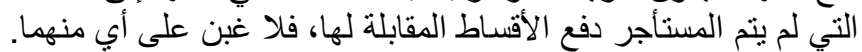

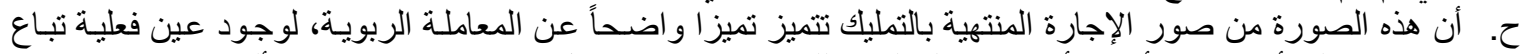

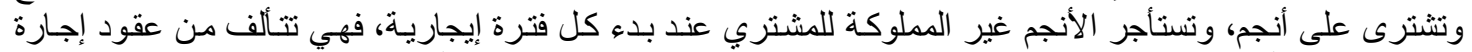

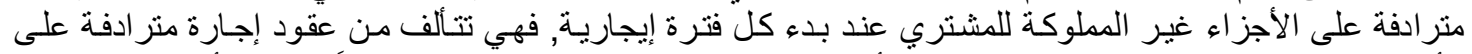

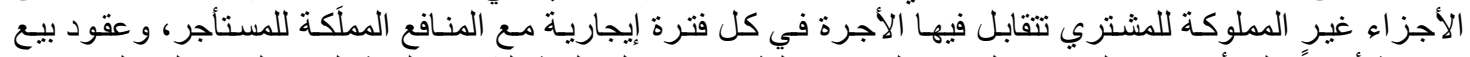

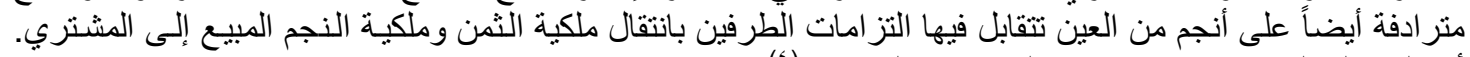

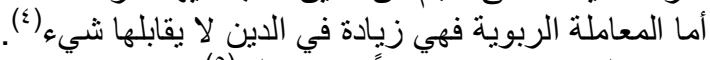

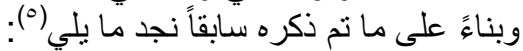

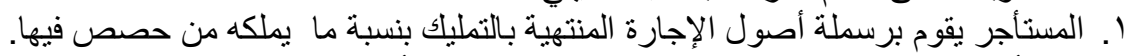

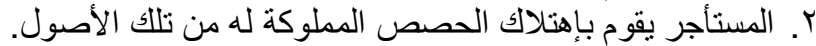

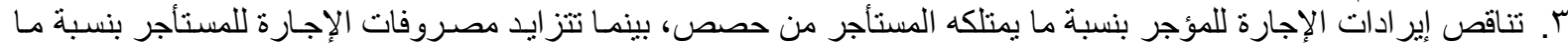

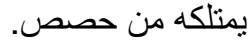

ع. توزع مصاريف الصيانة و الإصلاح لهذه الأصول على كل من المؤجر و المستأجر ونسبة حصص المكية المية لكل منهما.

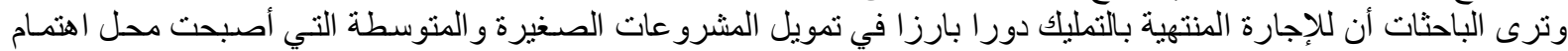

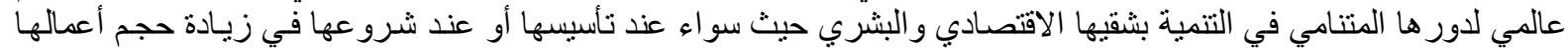

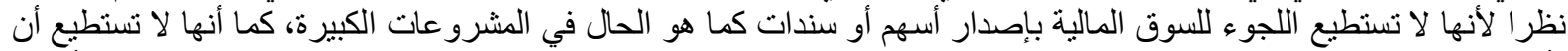

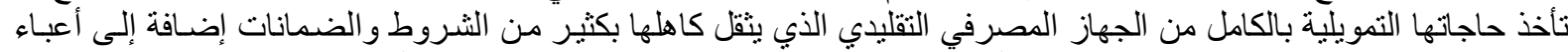

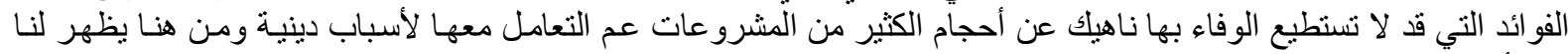

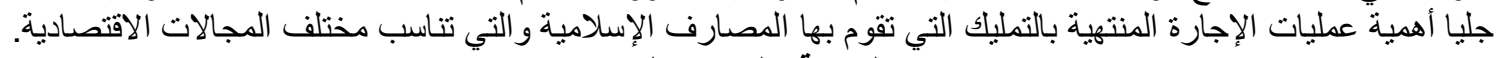
الخاتمة (النتائج والتوصيات)

توصلت الدراسة إلى عدد من الاستتناجات الآتية:

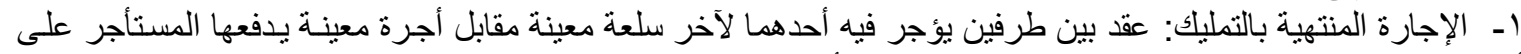

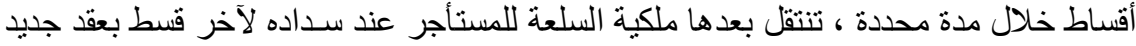

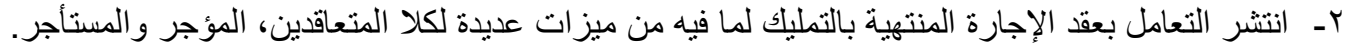

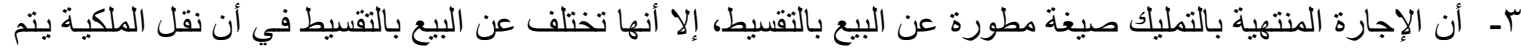
بعد انتهاء عقد الإجارة.

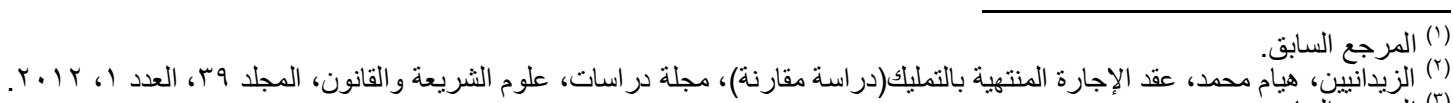

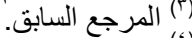
(§) انظرج المرجع السابق، السابق، القحف، الإجارة المنتهية بالتمليك، مرجع سابق، القرة داغي،علي محي الدين، الإيجار المنتهي بالتمليك،مجمع الفقه الإسلامي، 
عـ- موضوع عقد الإجارة المنتهية بالتمليك هو نقل المنفعة و العين، لكن نقل المنفعة بثكل يقيني، أمسا نقل العين فعلى وجـه

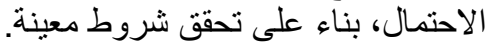
هـ - عقد الإجارة المنتهية بالتمليك هو عقد مركب من عن عدد من العقود، ونتيجـة لذلك ينتج تعقيد في الطبيعة القانونيـة لتلك العقود.

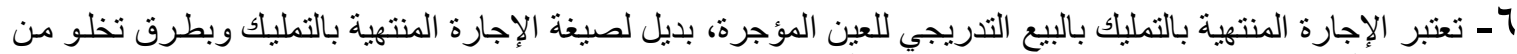

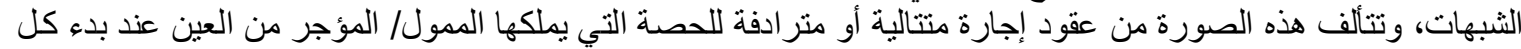

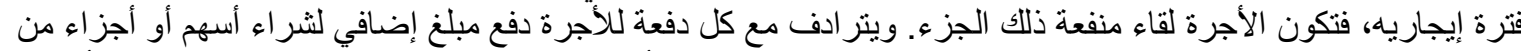

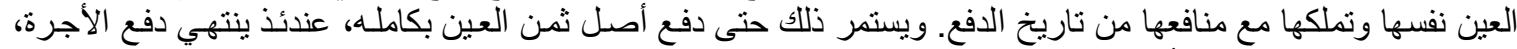
التوصيات

ويكون ذللك ضمن ضو ابط وأحكام الثريعة الإسلامية.

يوصى من خلال هذا البحث المتواضع إلى جملة من التوصيات الات بمكن تلخيصها بما يلي:

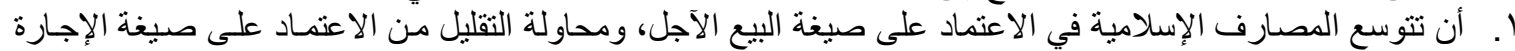

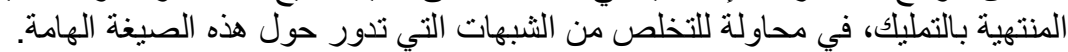

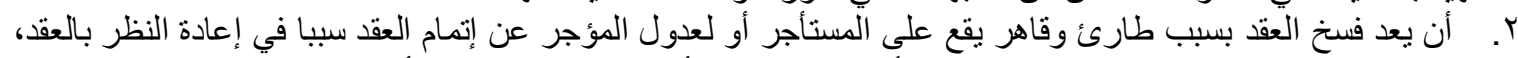

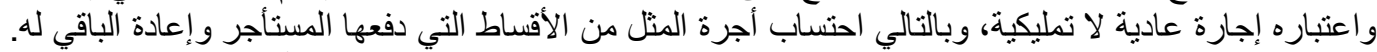

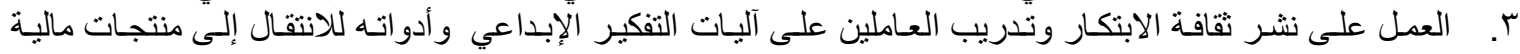

؛. تشجيع الأبحاث و الدر اسات وكل ما من شأنه تطوير المنتجات المالية الإسلامية.

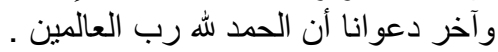

المراجع

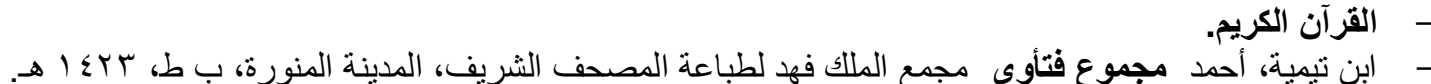

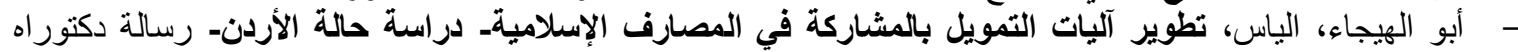

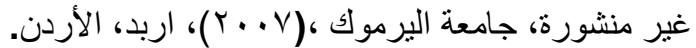

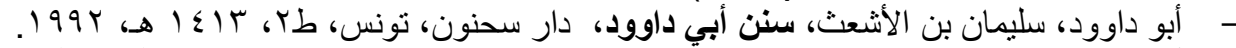

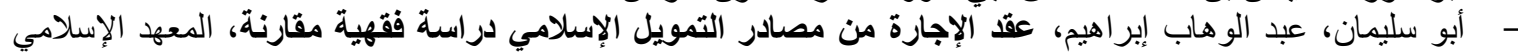

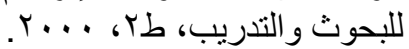

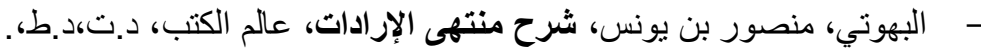

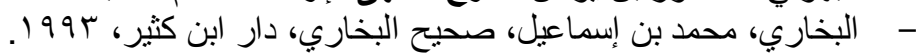

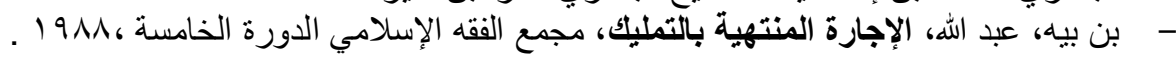

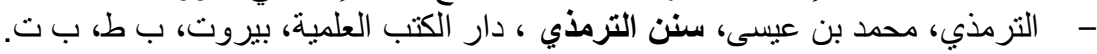

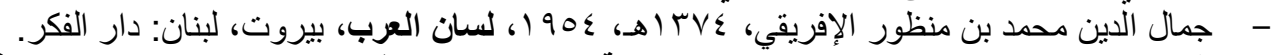

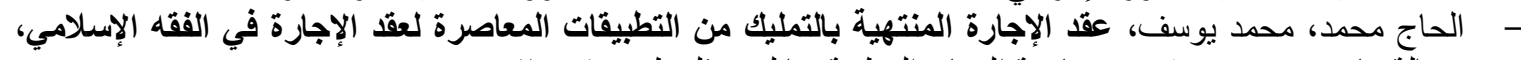

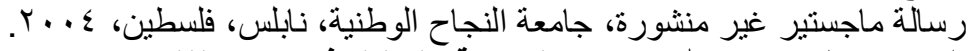

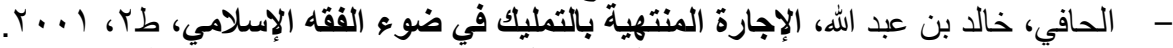

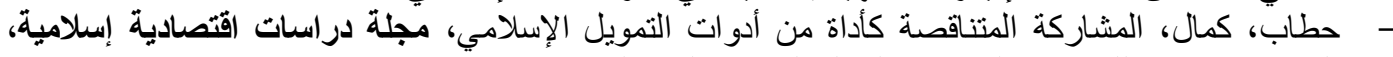

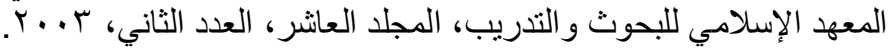

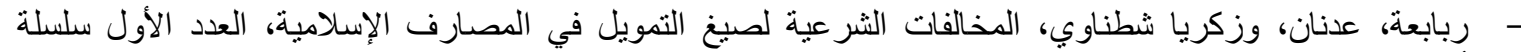

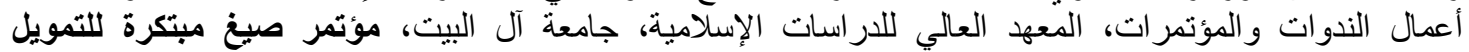

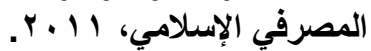
الرشيدي، محمد عبد الله، عقد الإجارة المنتهية بالتمليك ـ دراسة مقارنة مع الشريعة الإسلامية، رسالة ماجستير غير

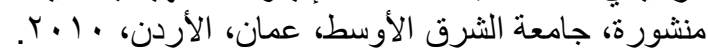

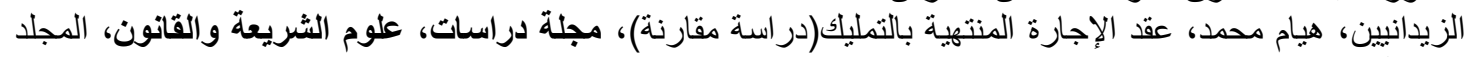

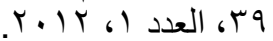

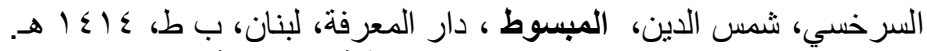
عبد الله، خالد، حسين سعيد، العمليات المصرفية الإسلامية ـ الطرق المعانية المحسبية الحليثة، طب، دار و ائل للنشر، عمان،

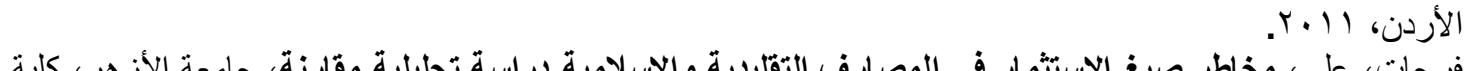
فرحات، علي، مخاطر صيغ الاستثمار في المصارف التقليدية والإسلامية دراسة تحليلية مقارنة، جامعة الأزهر، كلية

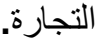

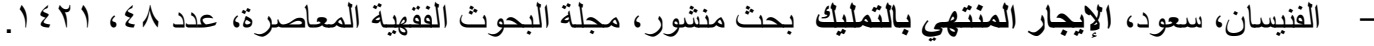




$$
\begin{aligned}
& \text { - - قحف، منذر، الإجارة المنتهية بالتمليك وصكوك الأعيان المؤجرة، دورة مجمع الفقه الإسلامي الدولي الثانية عشرة، }
\end{aligned}
$$

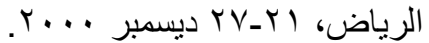

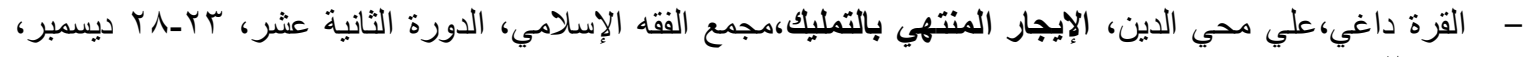

$$
\begin{aligned}
& \text {. .... } \\
& \text { - كامل، عمر عبد الله، "القواعد الفقهية الكبرى وأثرها في المعاملات المالية"، طبعة خاصة بدلة البركة، دار الكتبي، } \\
& \text { الجزء الثياني. } \\
& \text { - المعايير الثرعية لهيئة المحاسبة والمر اجعة للمؤسسات المالية المعاصرة. }
\end{aligned}
$$

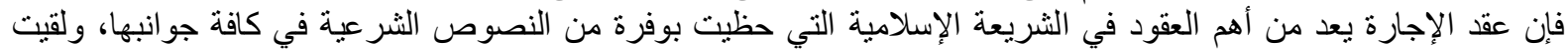

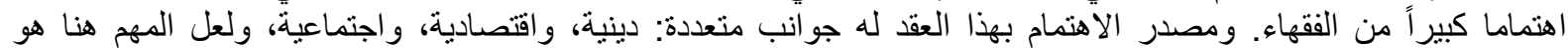

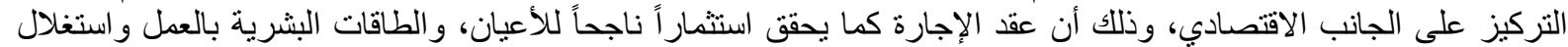

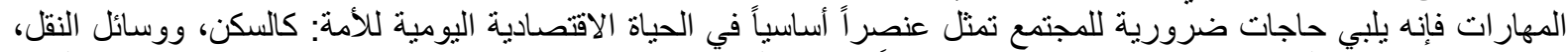

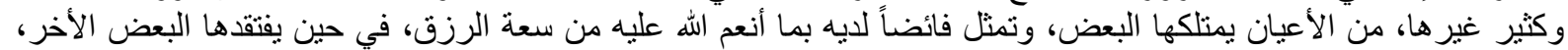

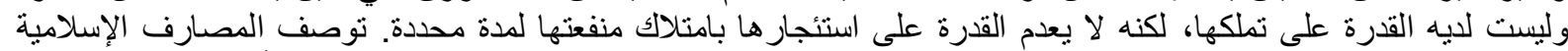

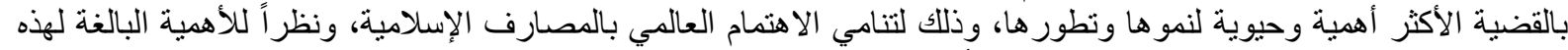

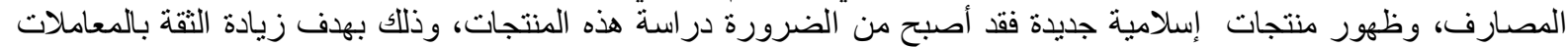

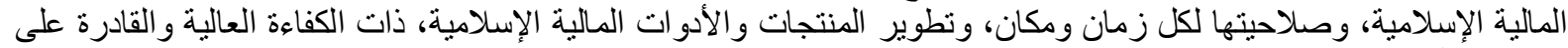

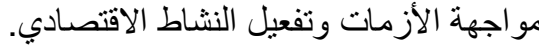

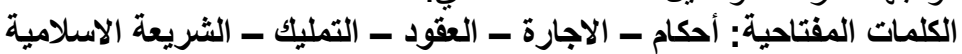

\section{Abstract}

The leasing contract is one of the most important contracts in Islamic law, which has received an abundance of legal texts in all its aspects, and has received great attention from jurists. The source of interest in this contract has multiple aspects: religious, economic, and social. Perhaps the most important thing here is to focus on the economic side, and that the leasing contract also achieves a successful investment for notables, and human energies to work and exploit skills, it meets the essential needs of society, which is an essential element in daily economic life For the nation: such as housing, means of transportation, and many others, of notables owned by some, and represents a surplus for him by the grace of God granted to him, while others lack it, and he does not have the ability to own it, but he does not execute the ability to rent it by owning its benefit for a specified period (). Islamic banks are described as the most important and vital issue for their growth and development, due to the growing global interest in Islamic banks, and given the extreme importance of these banks, and the emergence of new Islamic products, it has become necessary to study these products, with a view to increasing confidence in Islamic financial transactions, their suitability for every time and place, and product development And Islamic financial instruments, with high efficiency and able to face crises and activate economic activity.

Keywords: rule - rent - Agreement - Ownership - Islamic law 